\title{
Red blood cell distribution width is associated with mortality after acute ischemic stroke: a cohort study and systematic review
}

\author{
Lu Wang", Changyi Wang", Simiao Wu, Yuxiao Li, Wen Guo, Ming Liu \\ Center of Cerebrovascular Diseases, Department of Neurology, West China Hospital, Sichuan University, Chengdu 610041, China \\ Contributions: (I) Conception and design: M Liu; (II) Administrative support: M Liu; (III) Provision of study materials or patients: M Liu; (IV) \\ Collection and assembly of data: L Wang, C Wang; (V) Data analysis and interpretation: C Wang, Y Li, W Guo, L Wang; (VI) Manuscript writing: \\ All authors; (VII) Final approval of manuscript: All authors. \\ \#These authors contributed equally to this work. \\ Correspondence to: Ming Liu. Center of Cerebrovascular Diseases, Department of Neurology, West China Hospital, Sichuan University, No. 37 Guo \\ Xue Xiang, Chengdu 610041, China. Email: wyplmh@hotmail.com.
}

\begin{abstract}
Background: Whether red blood cell distribution width (RDW) is associated with the prognosis of acute ischemic stroke is inconclusive according to recent studies. We performed a cohort study and meta-analysis to explore the association between RDW and functional outcome.

Methods: Patients with ischemic stroke admitted to the Department of Neurology within 24 hours of stroke onset between January 1, 2015 to December 31, 2018 were enrolled. Blood was sampled within 24 hours after admission. We searched PubMed, Embase, Web of Science databases up to Nov 2019 to identify studies investigating the association between RDW values and prognosis following stroke. Outcomes included 3-month death and poor functional outcome [defined by modified Rankin Scale (mRS) score $\geq 3$ ].

Results: We included 1,558 patients in cohort study. RDW was independently associated with 3-month death [odds ratio (OR), 1.19; 95\% confidence interval (CI), 1.03, 1.37], but not associated with 3-month poor outcome (OR 1.05, 95\% CI, 0.95, 1.16), after adjustment for confounders. A dose-dependent relationship between RDW levels and 3-month death was revealed in the restricted cubic spline plot. Seven observational studies with 4,407 patients were identified for systematic review. When combining our study and previous studies, the association was significant for RDW predicting death (5 studies with 3,366 patients, OR 1.25, 95\% CI, 1.15, 1.35), as well as for poor outcome (4 studies with 3,483 patients, OR 1.23, 95\% CI, 1.05, 1.44). Conclusions: RDW was an independent predictor of 3-month functional outcome, and a trend of dosedependent relationship between RDW and 3-month death was detected.
\end{abstract}

Keywords: Red blood cells distribution width (RDW); acute ischemic stroke; death; disability

Submitted Aug 18, 2019. Accepted for publication Dec 17, 2019.

doi: $10.21037 /$ atm.2019.12.142

View this article at: http://dx.doi.org/10.21037/atm.2019.12.142

\section{Introduction}

Stroke is the leading cause of death and disability in the world. In China, the case fatality from hospital-based studies is less than $5 \%$ at 30 days, $10 \%$ at 90 days, and $15 \%$ at 1 year; about one third of patients will experience death or disability at 3 months and 1 year (1). According to American Heart Association, the mortality rate after stroke ranges from $10.5 \%$ at 30 days, $21.2 \%$ at 1 year, $39.8 \%$ at 5 years, up to $58.4 \%$ at the end of life $(2,3)$. Despite prognostic factors including age and the severity of stroke have been widespread acknowledged (4), the prognosis following stroke remains challenging to predict because of the complicated pathophysiological mechanisms behind stroke. Given the large stroke burden, new markers are needed to identify patients with poor outcome in order to accurately predict prognosis and optimize the administration of management. 
Recently, inflammation biomarkers in response to the brain tissue necrosis has sparked interest. Red blood cell distribution width (RDW) indicates the size variation in circulating erythrocyte, which is one of the biomarkers potentially involved in inflammation process, oxidative stress, and endothelial dysfunction in vascular diseases (5). The increased variation in the size of red blood cells (RBCs) is called anisocytosis. For many years, RDW has been applied to differentiate diagnosis of anemia in clinical setting. Recently, RDW has emerged as a biomarker in vascular diseases (5).

A descriptive review has suggested higher RDW independently predicts adverse outcome in patients with stroke (6). However, no uniform conclusions have been drawn about the role of RDW in the prognosis of ischemic stroke (7). One study has noted RDW higher than $14 \%$ is associate with an increased 1-year mortality of acute ischemic stroke (8). On the contrary, another study with 1,504 patients found RDW was not independently associated with functional outcome [modified Rankin Scale (mRS) score $\geq 3$ ] in multivariable analysis (9). An important limitation of these two previous studies was that neither of them reported numerical results of multivariate analysis, and thus the insufficient adjustment for potential confounders might weaken their power to determine the association between RDW and poor functional outcome.

Several potential mechanisms hint the relationship between RDW and prognosis after stroke. Firstly, an underlying inflammation and oxidative stress state might link increased RDW and poor clinical outcome (10). Secondly, increased RDW was inversely associated with oxygen saturation of blood. Previous evidence has suggested lower oxygen saturation (11) and reduced RBC deformability (12) in patients with higher RDW levels. The higher the RDW value, the lower the oxygen supply in tissues (13), which implies increased RDW levels may directly contribute to the development and worsening of stroke by reducing oxygenation in brain. Thirdly, previous studies have found that shorter telomere lengths and older age were significantly associated with increased RDW levels (14); a meta-analysis revealed that RDW is a powerful predictor of mortality in community-dwelling adults (15). Based on above-mentioned pieces of evidence, we aimed to investigate the association between RDW and functional outcome in acute ischemic stroke patients using data from our registry and from previously published literatures.

\section{Methods}

\section{Cobort study}

The retrospective cohort study was based on Chengdu Stroke Registry, which consecutively recruited stroke patients from 2002 in the Department of Neurology of West China Hospital, a 4,300-bed teaching hospital with about 5,000 patients discharged from the department annually. The project has been approved by the Scientific Research Department and conformed to Declaration of Helsinki. The detailed description of the registry has been reported elsewhere (16). We included patients diagnosed with acute ischemic stroke who were admitted from January 1, 2015 to December 31, 2018. The diagnosis of acute ischemic stroke was made in accord with the World Health Organization (WHO) definition as rapidly developing focal or global neurologic impairment, with symptoms lasting at least 24 hours or leading to death, and with no other apparent origin expect for cerebral vessels (17). The inclusion criteria were older than 18 years old and arrival at hospital within 24 hours from symptom onset. We excluded patients with transient ischemic attack or hemorrhagic stroke, patients with insufficient data in laboratory test, and patients without follow up information.

\section{RDW measurement}

Blood samples were collected from a peripheral vein using ethylene diamine tetraacetic acid (EDTA) tube within 24 hours after admission and analyzed using the Sysmex automated hematology analyzer (Sysmex, Kobe, Japan). Two parameters of RDW were calculated to measure the extent of anisocytosis: the standard deviation (SD) and the coefficient variation (CV). RDW-CV is widely investigated and is calculated according to the following formula: RDW$\mathrm{CV}=(\mathrm{SD}$ of erythrocyte volume/mean corpuscular volume) $\times 100$. The reference range of RDW-CV is $11.5-14.5 \%$ (18). In this study, we reported RDW-CV and used RDW to represent it.

\section{Data collection}

We recorded patients' information by structured forms including: demographic statistic data, admission National Institute of Health Stroke Scale (NIHSS) score, cerebrovascular risk factors, Trial of ORG 10172 in Acute Stroke Treatment (TOAST) classification, in-hospital treatment, in-hospital complications, and clinical outcome. We defined NIHSS score higher than 15 as severe stroke, 
and below 15 as mild stroke. Anemia was diagnosed when the level of hemoglobin was less than $120 \mathrm{~g} / \mathrm{L}$ in men and $110 \mathrm{~g} / \mathrm{L}$ in women by Chinese criteria (19). Infection was diagnosed by the treating physician, based on medical records including symptoms and signs, laboratory tests, antibiotic treatment, as well as chest computed tomography scans. All patients were treated according to treatment guideline and patients eligible for reperfusion therapy were treated with intravenous thrombolysis or endovascular therapy.

\section{Outcome assessment}

Our primary outcome was death within 3 months following admission. Our secondary outcome was 3 -month poor outcome, defined by mRS score from 3 to 6. Patients were followed up at 3-month by telephone interview.

\section{Statistical analysis}

We categorized patients to three groups according to RDW levels. If variables were normal distribution, they were reported as mean and $\mathrm{SD}$, otherwise, they were presented as median and interquartile range (IQR). Difference of continuous variables was compared by one-way ANOVA and Kruskal-Wallis $\mathrm{H}$ test. Categorical variables were expressed as counts and percentages and compared with the $\chi^{2}$ test or Fisher exact tests. We calculated spearman correlation coefficients between RDW values and age, NIHSS score, hemoglobin, platelet counts, white blood cells (WBC), international normalized ratio (INR), glucose, creatinine, and onset to admission time to determine correlations between RDW and these variables.

For the multivariable logistic regression analyses, we included variables with $\mathrm{P}$ value $<0.05$ in univariate analysis. In model 1, we adjusted for age and sex; in model 2, we further adjusted for other confounders. Odds ratios (ORs) and $95 \%$ confidence intervals (CIs) were reported both in univariable and multivariable analyses. We performed tests for linear trend ( $\mathrm{P}$ for trend) by entering the median RDW value in each group as a continuous variable in the models. We also evaluated the pattern and magnitude of the association between the RDW and outcome using a multivariable logistic regression model with restricted cubic splines at 4 knots: the 25 th, 50 th, 75 th, and 95 th percentiles. To determine discriminative ability of RDW, we generated a receiver operating characteristic (ROC) curve and calculated the sensitivity and specificity of RDW values to predict 3-month outcome.

We performed subgroup analyses stratified by variables including age ( $\leq 65$ and $>65)$, stroke severity (mild stroke and severe stroke), accompanying with anemia or atrial fibrillation, treated with reperfusion therapy, developing post-stroke infection or not. The difference between subgroup were inspected by interaction analysis using the likelihood ratio tests. We performed statistical analyses by SPSS version 21.0 (IBM Corporation, Armonk, NY, USA) and Stata Version 15.0 (College Station, TX, USA). A two side $\mathrm{P}<0.05$ was statistically significant.

\section{Systematic review}

We conducted literature search to identify studies reporting the association between RDW values and prognosis after acute ischemic stroke using terms combining RDW and acute ischemic stroke with their synonyms. The search was performed in PubMed, Embase, and Web of Science databases up to Nov 2019. Studies were eligible if they (I) recruited patients with acute ischemic stroke; (II) investigated the association between RDW levels and functional outcome (evaluated by $\mathrm{mRS}$ score). We extracted data of sample size, mean age, sex, onset to admission time, mean RDW value, and patient number of poor outcome from each included study. A meta-analysis pooling our cohort data and previous published data were performed. Fixed effects model was used if heterogeneity was low $\left(\mathrm{I}^{2}<50 \%\right)$. Otherwise, random effects model was applied. We drew the funnel plot to observe the symmetry of plot and performed Egger test to identify publication bias. We reported registry study according to the Strengthening the Reporting of Observational Studies in Epidemiology guidelines (20) and described systematic review on the basis of the Meta-analysis Of Observational Studies in Epidemiology guidelines (21).

\section{Results}

\section{Cobort study}

Of 1,700 patients eligible from Chengdu Stroke Registry, 142 patients were excluded because of unavailable laboratory tests or missing follow-up information (Figure S1). In total, 1,558 patients were included ( 838 men and 720 women; mean age $66.20 \pm 13.94$ years, median/IQR of NIHSS score was $6 / 2$ 12). The median/IQR time of RDW analysis since stroke onset was 10/4-24 hours. The mean RDW value was $13.8 \%$ (median/IQR: $13.60 \% / 13.0-14.3 \%$ ) and the low, medium, and high tertiles distribution were $\mathrm{T}_{1}<13.0 \%, \mathrm{~T}_{2}=13.0$ 
$13.9 \%, \mathrm{~T}_{3} \geq 14.0 \%$, respectively. At 3 months follow-up, 611 (39.44\%) patients developed poor functional outcome and $130(8.34 \%)$ deaths were reported. The incidence of death increased in parallel with the increment of RDW levels from $4.34 \%$ in $\mathrm{T}_{1}, 8.19 \%$ in $\mathrm{T}_{2}$, to $10.97 \%$ in $\mathrm{T}_{3}(\mathrm{P}=0.002)$. Additionally, the higher the RDW level, the larger the mRS score, and the patients with RDW levels $\geq 14.0 \%$ had higher proportion of poor functional outcome (Figure S2, $\mathrm{P}=0.001$ ).

Patients with higher RDW were older, had lower level of hemoglobin, and higher level of INR and creatinine. Patients in higher RDW groups had higher prevalence of atrial fibrillation, rheumatoid heart disease, coronary heart disease, and cardio-embolism stroke $(\mathrm{P}<0.05$, Table 1$)$. RDW positively correlated with age $(\mathrm{r}=0.174$, $\mathrm{P}<0.001)$, NIHSS score $(\mathrm{r}=0.108, \mathrm{P}<0.001)$, INR $(\mathrm{r}=0.182$, $\mathrm{P}<0.001)$, creatinine $(\mathrm{r}=0.131, \mathrm{P}<0.001)$, and inversely correlated with hemoglobin $(\mathrm{r}=-0.267, \mathrm{P}<0.001)$, glucose $(\mathrm{r}=-0.080, \mathrm{P}=0.0018)$, platelet counts $(\mathrm{r}=-0.106, \mathrm{P}<0.001)$. No correlation was observed between RDW and WBC $(\mathrm{r}=0.012, \mathrm{P}=0.629)$, as well as onset to admission time $(\mathrm{r}=0.0001, \mathrm{P}=0.998)$.

The comparison of baseline characteristics between survivors and non-survivors is presented in Table S1. The median RDW was significantly higher in non-survivors, compared with survivors $(14.27 \%$ versus $13.78 \%, \mathrm{P}<0.001)$. Significant differences in age, NIHSS score, atrial fibrillation, TOAST classification, hemoglobin, WBC, glucose, creatinine, anticoagulants, and in-hospital infection were observed between patients who survived and those who died at 3-month. These potential confounding factors were adjusted in multivariable analysis to identify the independent association between RDW and outcome.

When RDW was regarded as a continuous variable, univariable and multivariable analysis showed that increased RDW was associated with an enhanced rate of 3-month death. RDW per $1 \%$ increment increased the risk of 3 -month death by $23 \%$ after adjusting for age and sex (model 1), and by $19 \%$ after further adjusting for NIHSS score, atrial fibrillation, rheumatoid heart disease, coronary heart disease, current drinking, TOAST classification, creatinine, WBC, glucose, reperfusion therapy, antiplatelets, anticoagulants, in-hospital infection, and anemia (OR 1.19, 95\% CI, 1.03, 1.37, $\mathrm{P}=0.018$, Table 2). The adjusted OR (95\% CI; P value) for the medium and the highest tertile versus the lowest tertile was 2.35 (1.15-4.82; 0.020), 2.52 $(1.23-5.16 ; 0.011)$, respectively $\left(\mathrm{P}_{\text {for trend }}=0.042\right)$. Figure 1 demonstrates a trend of dose-dependent relationship between RDW levels and 3-month death, where 14\% was the reference value for RDW. The magnitude of the association (adjusted OR for 3-month death) increased along with the increment of RDW levels.

Based on the ROC curve, RDW values for predicting 3 -month death yielded an area under the curve (AUC) of 0.620 (95\% CI, 0.572-0.668; Figure S3). The optimal RDW cutoff for predicting 3-month death was $13.3 \%$, with sensitivity of $80 \%$ and specificity of $38 \%$. No significant interaction between RDW and covariates was revealed (all $\mathrm{P}_{\text {for interaction }}>0.05$, Figure 2).

When RDW was tested as a continuous variable, the crude OR for 3-month poor outcome was 1.17 , 95\% CI (1.08-1.27), $\mathrm{P}<0.001$. However, after fully adjusting for potential confounding factors in model 2 , no significant association between RDW and 3-month poor outcome was discovered: for RDW as a continuous variable, adjusted OR $1.05,95 \%$ CI $(0.95,1.16)$ and for $\mathrm{RDW} \geq 14.0 \%$, adjusted OR $0.89,95 \%$ CI $(0.63,1.24)$ (Table 2). Figure 1 shows the fluctuant change of OR along with the increment of RDW levels: RDW $<14 \%$ and RDW $>16 \%$ were associated with an increased OR for 3-month poor outcome, while RDW level from $14 \%$ to $16 \%$ was associated with a reduced OR.

\section{Systematic review}

We retrieved 201 studies and excluded 194 records due to following reasons: 93 irrelevant studies, 60 duplicate records, 18 abstracts, 12 studies reported the association between RDW and the risk of stroke, 4 reviews, 7 studies included patients with transient ischemic attack or hemorrhagic stroke (Figure S4). Finally, 7 cohort studies involving 4,407 patients were included $(6-9,13,22,23)$. Table 3 shows the basic characteristics of included studies. The sample-size weighted mortality was $10.3 \%$, and sample-size weighted rate of poor outcome was $43.3 \%$; the sample-size weighted mean RDW value was $13.74 \%$.

One study did not provide numerical results of OR and $95 \%$ CI, which reported no significant association between RDW and functional outcome after adjusting cofounding factors (9). Six studies indicated that RDW was independently associated with poor outcome or death after stroke $(6-8,13,22,23)$. Figure 3 summarized the meta-analysis results pooled by random effects model. Five studies incorporating 3,366 patients reported the association between RDW and death: pooled OR 1.25, 95\% CI $(1.15,1.35)$ with low heterogeneity $\left(\mathrm{I}^{2}=37.1 \%\right)$; and for predicting poor outcome, pooled OR of 4 studies involving 
Table 1 Baseline characteristics of included patients grouped by tertile of red cell distribution width

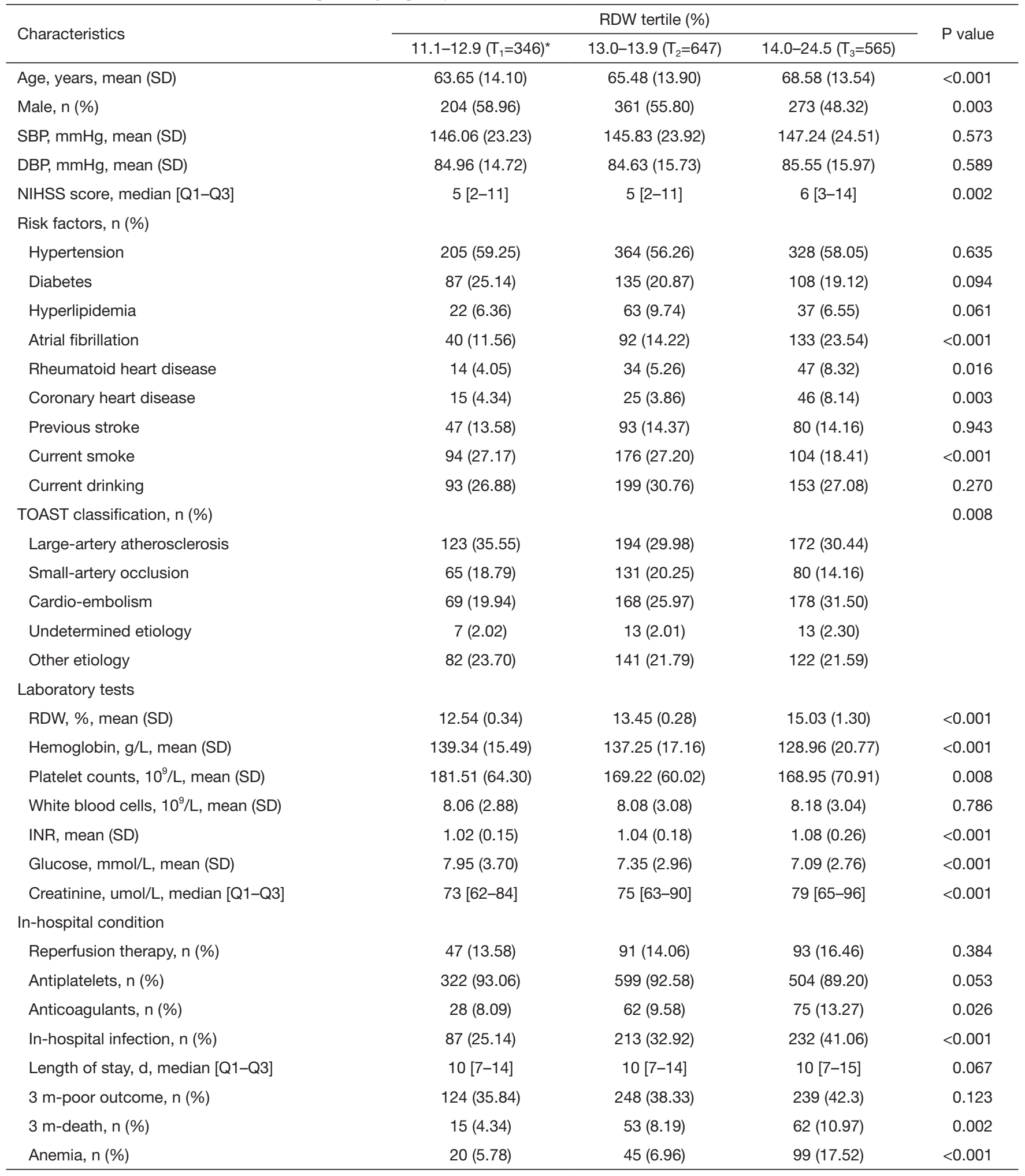

*, the range of RDW and the number of patients in each tertile. RDW, red cell distribution width; T, tertile; SBP, systaltic blood pressure; DBP, diastolic blood pressure; NIHSS, National Institute of Health Stroke Scale; INR, International Normalized Ratio; TOAST, Trial of ORG 10172 in Acute Stroke Treatment (TOAST) classification; m, month. 
Table 2 Logistic regression analysis for 3-month death and 3-month poor outcome

\begin{tabular}{|c|c|c|c|}
\hline & $\begin{array}{l}\text { Univariate analysis, } \\
\text { OR (95\% Cl), P value }\end{array}$ & \multicolumn{2}{|c|}{ Multivariate analysis, OR (95\% Cl), P value } \\
\hline \multicolumn{4}{|l|}{ 3-month death } \\
\hline RDW (per 1\% increment) & $1.26(1.13,1.41),<0.001$ & $1.23(1.10,1.39),<0.001$ & $1.19(1.03,1.37), 0.018$ \\
\hline \multicolumn{4}{|l|}{ RDW tertile } \\
\hline $\mathrm{T} 2$ & $1.97(1.09,3.55), 0.024$ & $1.86(1.03,3.36), 0.040$ & $2.35(1.15,4.82), 0.020$ \\
\hline T3 & $2.72(1.52,4.86),<0.001$ & $2.31(1.29,4.16), 0.005$ & $2.52(1.23,5.16), 0.011$ \\
\hline$P$ for trend & $<0.001$ & 0.008 & 0.042 \\
\hline \multicolumn{4}{|l|}{ 3-month poor outcome } \\
\hline $\mathrm{T} 1$ & Reference & Reference & Reference \\
\hline $\mathrm{T} 2$ & $1.12(0.86,1.47), 0.406$ & 1.06 (0.81, 1.40), 0.667 & $1.05(0.76,1.45), 0.754$ \\
\hline T3 & $1.32(1.00,1.74), 0.050$ & $1.14(0.86,1.52), 0.355$ & $0.89(0.63,1.24), 0.487$ \\
\hline$P$ for trend & 0.041 & 0.343 & 0.351 \\
\hline
\end{tabular}

Model 1: adjusted for age and sex. Model 2: adjusted for age, sex, NIHSS scores, atrial fibrillation, rheumatoid heart disease, coronary heart disease, current drinking, TOAST classification, creatinine, white blood cells, glucose, reperfusion therapy, antiplatelets, anticoagulants, in-hospital infection, anemia. RDW, red cell distribution width; T, tertile; NIHSS, National Institutes of Health Stroke Scale; OR, odds ratio; 95\% Cl, 95\% confidence interval; TOAST, Trial of ORG 10172 in Acute Stroke Treatment (TOAST) classification; m, month.
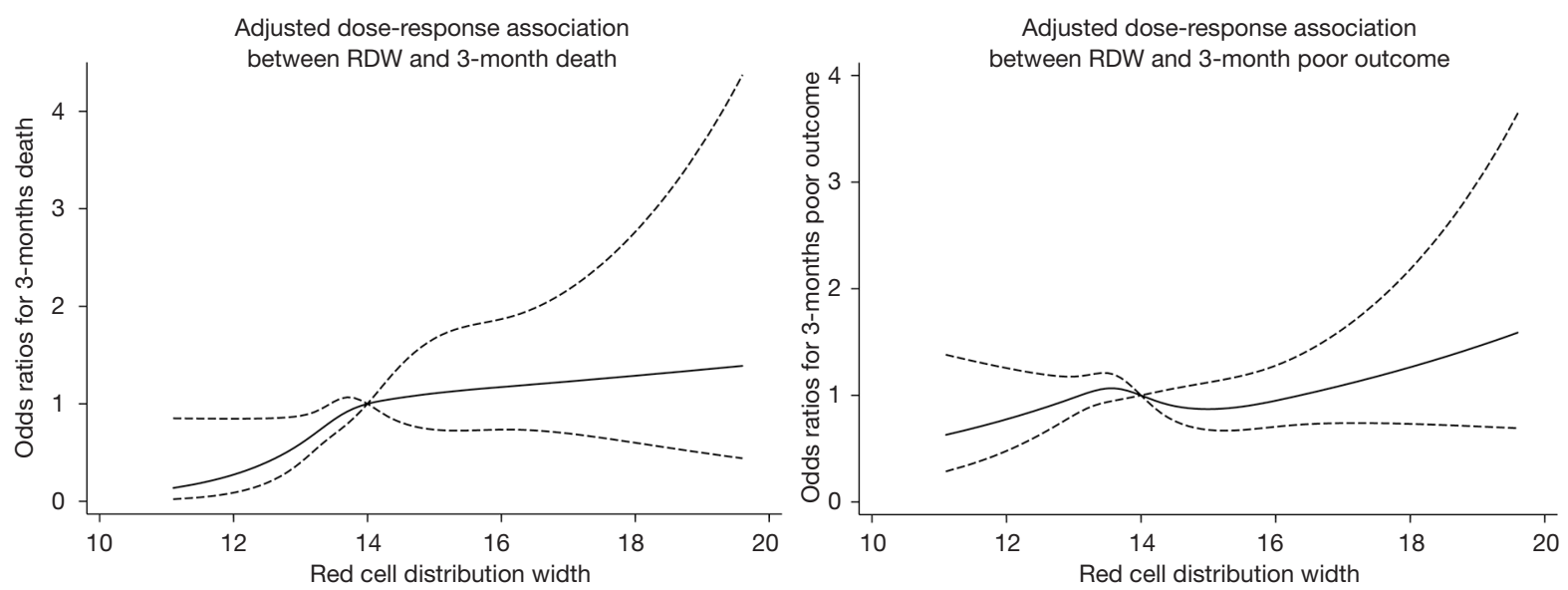

Figure 1 Adjusted dose-response association between red cell distribution width (RDW) values (\%) and 3-month death (left) and 3-month poor outcome (right). Red cell distribution width (RDW) was coded using a restricted cubic spline function with four knots located at the 25 th, 50th, 75th, and 95th percentiles of the distribution of RDW. Reference value is 14\% for RDW. Y-axis represents the adjusted odds ratios of outcomes when comparing individuals with any value of RDW with individuals with the reference value of RDW (14\%). Dashed lines are $95 \%$ confidence intervals. Adjusted factors included age, sex, NIHSS score, atrial fibrillation, rheumatoid heart disease, coronary heart disease, current drinking, TOAST classification, creatinine, white blood cells, glucose, reperfusion therapy, antiplatelets, anticoagulants, inhospital infection, anemia. 


\begin{tabular}{|c|c|c|c|}
\hline Subgroup analysis & Odds ratio $(95 \%$ & nce interval), $\mathrm{p}$-value* & p-value for interaction \\
\hline Age & & & 0.212 \\
\hline$\leqslant 65$ & •— & $1.08(0.82,1.43), 0.589$ & \\
\hline$>65$ & $1-1$ & $1.27(1.06,1.54), 0.012$ & \\
\hline Stroke severity & & & 0.474 \\
\hline mild stroke & & $1.04(0.83,1.30), 0.720$ & \\
\hline severe stroke & 1 & $1.30(1.05,1.61), 0.014$ & \\
\hline Anemia & & & 0.231 \\
\hline no & $=-1$ & $1.24(1.04,1.47), 0.018$ & \\
\hline yes & & $0.97(0.71,1.32), 0.847$ & \\
\hline Atrial fibrillation & & & 0.169 \\
\hline no & $=-1$ & $1.12(0.95,1.33), 0.185$ & \\
\hline yes & & $1.48(1.04,2.11), 0.028$ & \\
\hline Reperfusion therapy & & & 0.422 \\
\hline no & $\mapsto$ & $1.27(1.07,1.50), 0.006$ & \\
\hline yes & 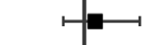 & $1.11(0.80,1.53), 0.530$ & \\
\hline Post-stroke infection & & & 0.135 \\
\hline no & 1 & $1.03(0.73,1.46), 0.851$ & \\
\hline yes & - & $1.27(1.07,1.51), 0.006$ & \\
\hline
\end{tabular}

Figure 2 Stratified logistic regression analysis to identify variables that modify the correlation between red cell distribution width (RDW) values and 3-month death. Adjusted factors included age, sex, NIHSS score, atrial fibrillation, rheumatoid heart disease, coronary heart disease, current drinking, TOAST classification, creatinine, white blood cells, glucose, reperfusion therapy, antiplatelets, anticoagulants, inhospital infection, anemia. The model was not adjusted for the stratification variable in each stratified analysis. $\mathrm{P}$ value* indicates the $\mathrm{P}$ value for the corresponding odds ratio of each subgroup.

Table 3 Baseline characteristics of included patients in systematic review

\begin{tabular}{|c|c|c|c|c|c|c|c|}
\hline Study & Place & $\begin{array}{l}\text { Sample } \\
\text { size }\end{array}$ & $\begin{array}{l}\text { Mean } \\
\text { Age }\end{array}$ & $\begin{array}{l}\text { Male } \\
(\%)\end{array}$ & $\begin{array}{l}\text { Onset to } \\
\text { admission time (h) }\end{array}$ & $\begin{array}{l}\text { Mean RDW } \\
\text { value }\end{array}$ & Number of poor outcome (\%) \\
\hline Ntaios [2011] & Switzerland & 1,504 & 72 & 56.1 & 24 & 14.1 & NA \\
\hline Kim [2012] & & & & & & & 3 m poor outcome: $186 / 830(22.4 \%)$ \\
\hline Duchnowski [2017] & Poland & 14 & NA & NA & NA & 14 & 1 m death: $4 / 14(28.6 \%)$ \\
\hline \multirow[t]{2}{*}{ Pinho [2018] } & Portugal & 602 & 74 & 42.7 & 4.5 & 14 & 1 y death: $117 / 591(19.8 \%)$ \\
\hline & & & & & & & 3 m poor outcome: $307 / 558$ (55\%) \\
\hline Turcato [2017] & Italy & 837 & 77 & NA & 12 & 13.9 & 3 m death: 70/837 (8.4\%); \\
\hline Present study & & & & & & & 3 m poor outcome: $611 / 1,558(39.44 \%)$ \\
\hline
\end{tabular}

RDW, red cell distribution width; $m$, month; $y$, year. 


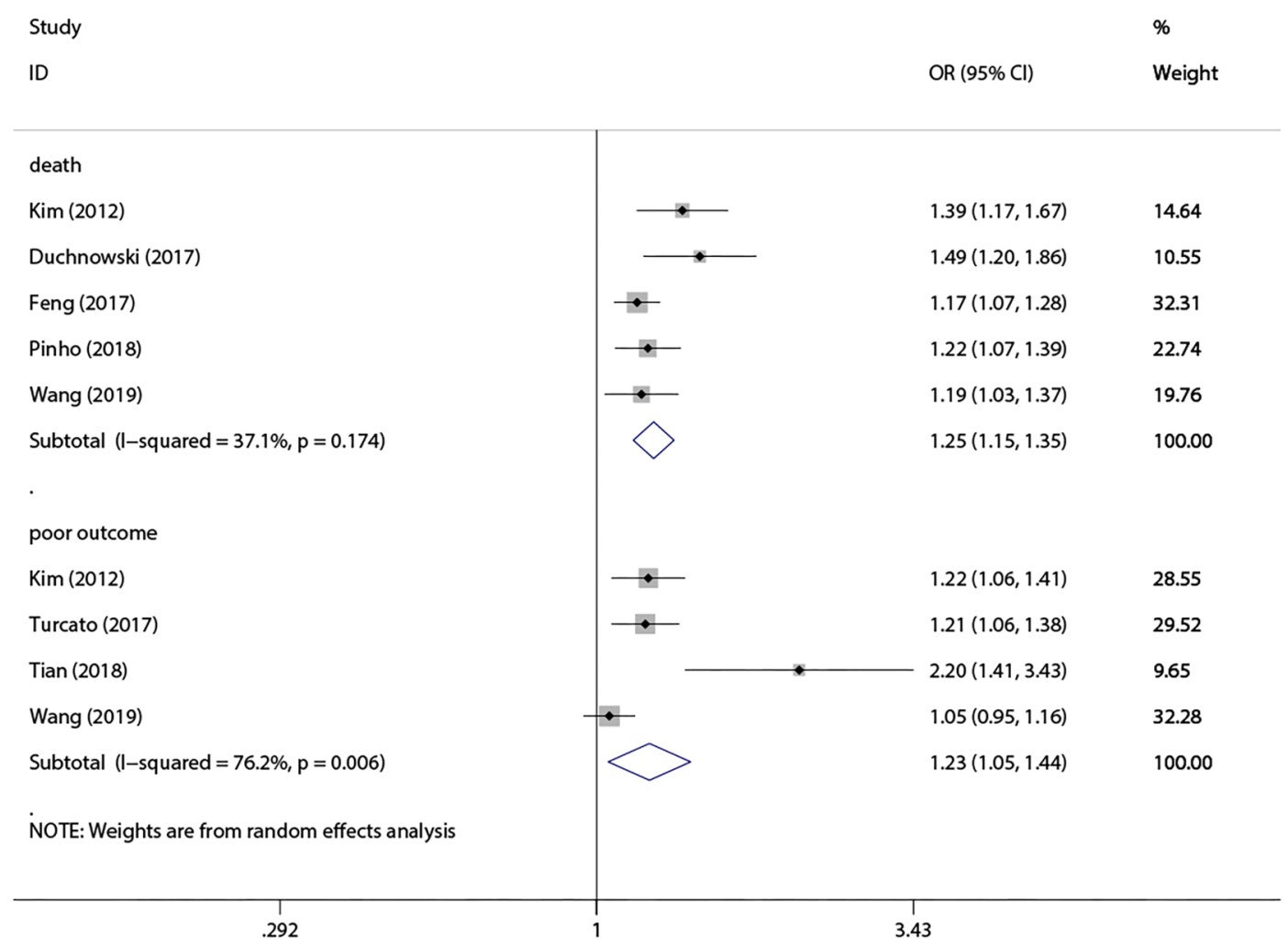

Figure 3 Forest plot of odds ratios (OR) and 95\% confidence intervals (CIs) for red cell distribution width (RDW) predicting death and poor outcome pooling our cohort data and previous published data.

3,483 patients was $1.23,95 \%$ CI $(1.05,1.44)$ with medium heterogeneity $\left(\mathrm{I}^{2}=76.2 \%\right)$. The asymmetric funnel plot indicated the existence of publication bias (Figure S5). $\mathrm{P}$ value of Egger test was 0.031 for predicting death and 0.035 for predicting poor outcome.

\section{Discussion}

To provide additional evidence on the potential association of baseline RDW with prognosis following acute ischemic stroke, we performed a cohort study and systematically reviewed current literature. We found RDW was an independent predictor of 3-month death, and a trend of dose-dependent relationship was detected. Meta-analysis of our study and previous studies suggested RDW was associated with both death and poor outcome after stroke. Inconsistent with meta-analysis, we did not observe significant association between RDW and poor outcome in our registry data. Although it was unclear why our study was not in line with most prior studies, considering the medium heterogeneity in the analysis, difference in patient characteristics such as age, stroke severity, and the time of blood collection may partly explain the inconsistence.

Kara has revealed that elevated RDW could independently predict stroke severity assessed by neurological scoring systems (24). A literature review has suggested higher RDW was associated with adverse outcome in patients with ischemic stroke and cerebral embolism (6). Their findings were in accord with ours and provided support for our present study.

It is still unknow why RDW associated with outcome, but there are several plausible explanations about the underlying biological mechanisms. The correlation between RDW and inflammatory markers such as C-reactive protein has been reported (25). The inflammation and oxidative stress may disturb erythropoiesis, which may inhibit the maturation of RBCs, result in anisocytosis, and lead to higher RDW in patients. Besides, elevated RDW may reflect malnutrition and other disease, which may all related to the prognosis of stroke patients (26). Secondly, patients 
with higher RDW levels may have decreased oxygen saturation of blood and poor physiologic reserve to protect against hypoxic stress (27), the ischemic cerebral tissue may become necrosis without adequate oxygen supply. Thirdly, patients with higher RDW may be more likely to have abnormal coagulate function and higher risk of thromboembolism (28), which may lead to a greater chance of recurrent stroke, and more severe brain damage, and finally result in poorer outcome.

Our finding confirmed the prognostic value of RDW in patients with acute ischemic stroke and extended our knowledge regarding the potential dose-dependent relationship between RDW and death. As far as we know, the present cohort study had the largest sample size among previously published studies, comprehensively collected information about potential confounders, sufficiently adjusted them in the multivariate models, and quantitatively pooled all the evidence up to now. However, it is poorly understood whether increased RDW is a cause of, or just a bystander to poor outcome following stroke. RDW is significantly associated with many diseases related to thrombotic and inflammatory conditions, including atrial fibrillation, venous thromboembolism, and myocardial infarction (29-31). Moreover, RDW may serve as a prognostic marker in many cardiac surgeries $(32,33)$. Considering the predictive role of RDW in many other diseases, RDW is more likely a general marker rather than a stroke-specific predictor. Future studies may explore whether application of RDW could help doctors make decisions when assigning optimal therapeutic regimen and stratifying risk in secondary prevention in their clinical practice.

However, some limitations should be considered. Firstly, publication bias may exist. Generally, studies with positive results are more likely to be published and studies with negative results usually did not report OR and $95 \%$ CI. Therefore, we could not include the numerical results of these studies into the quantitative analysis and a definite conclusion could not be drawn. The funnel plot also suggested the existence of publication bias. More highquality studies are needed to investigate the value of RDW on prognosis after stroke. Secondly, the relationship between RDW and poor outcome needs further investigation. Although our results revealed significant association between RDW and death, the association between RDW and poor functional outcome was inconsistent. This was supported by the study of Pinho (8); they found although patients with higher RDW quartiles had higher rate of poor functional outcome, the difference was not significant after excluding patients died during the first 3 months. It seems that the prognostic value of RDW is stronger for death than any other outcome indicators, indicating that elevated RDW may mainly affect mortality. Indeed, there were much more evidence about the association of RDW and mortality than disability $(34,35)$. Thirdly, the role of RDW in the reoccurrence of vascular disease is still unclear. Previous studies have demonstrated increased risk of stroke and coronary heart disease in patients with higher RDW values $(36,37)$. Whether the association between RDW and mortality is due to higher risk of recurrent vascular diseases or not remains to be clarified.

\section{Conclusions}

RDW was an independent predictor of 3 -month functional outcome, and a trend of dose-dependent relationship between RDW and 3-month death was detected.

\section{Acknowledgments}

Funding: This study was funded by Key Research and Development Program, Science and Technology Department of Sichuan Province (Grant No. 2017SZ0007) and Major International (Regional) Joint Research Project, National Natural Science Foundation of China (Grant No. 81620108009).

\section{Footnote}

Conflicts of Interest: The authors have no conflicts of interest to declare.

Ethical Statement: The authors are accountable for all aspects of the work in ensuring that questions related to the accuracy or integrity of any part of the work are appropriately investigated and resolved. Ethics approval is obtained from the Biomedical Research Ethics Committee of West China Hospital, Sichuan University (Reference No. 2017130). Since all laboratory data were collected anonymously based on routine clinical setting and the study is a retrospective cohort, the ethics committee waived the need for consent.

\section{References}

1. Wu S, Wu B, Liu M, et al. Stroke in China: advances and 
challenges in epidemiology, prevention, and management. Lancet Neurol 2019;18:394-405.

2. Mozaffarian D, Benjamin EJ, Go AS, et al. Heart Disease and Stroke Statistics-2016 Update: A Report From the American Heart Association. Circulation 2016;133:e38-360.

3. Turcato G, Cappellari M, Follador L, et al. Red Blood Cell Distribution Width Is an Independent Predictor of Outcome in Patients Undergoing Thrombolysis for Ischemic Stroke. Semin Thromb Hemost 2017;43:30-5.

4. Weimar C, Konig IR, Kraywinkel K, et al. Age and National Institutes of Health Stroke Scale Score within 6 hours after onset are accurate predictors of outcome after cerebral ischemia: development and external validation of prognostic models. Stroke 2004;35:158-62.

5. Danese E, Lippi G, Montagnana M. Red blood cell distribution width and cardiovascular diseases. J Thorac Dis 2015;7:E402-11.

6. Feng GH, Li HP, Li QL, et al. Red blood cell distribution width and ischaemic stroke. Stroke Vasc Neurol 2017;2:172-5.

7. Kim J, Kim YD, Song TJ, et al. Red blood cell distribution width is associated with poor clinical outcome in acute cerebral infarction. Thromb Haemost 2012;108:349-56.

8. Pinho J, Marques SA, Freitas E, et al. Red cell distribution width as a predictor of 1-year survival in ischemic stroke patients treated with intravenous thrombolysis. Thromb Res 2018;164:4-8.

9. Ntaios G, Gurer O, Faouzi M, et al. Red cell distribution width does not predict stroke severity or functional outcome. Int J Stroke 2012;7:2-6.

10. Salvagno GL, Sanchis-Gomar F, Picanza A, et al. Red blood cell distribution width: A simple parameter with multiple clinical applications. Crit Rev Clin Lab Sci 2015;52:86-105.

11. Tomkiewicz-Pajak L, Plazak W, Kolcz J, et al. Iron deficiency and hematological changes in adult patients after Fontan operation. J Cardiol 2014;64:384-9.

12. Patel KV, Mohanty JG, Kanapuru B, et al. Association of the red cell distribution width with red blood cell deformability. Adv Exp Med Biol 2013;765:211-6.

13. Turcato G, Cervellin G, Cappellari M, et al. Early function decline after ischemic stroke can be predicted by a nomogram based on age, use of thrombolysis, RDW and NIHSS score at admission. J Thromb Thrombolysis 2017;43:394-400.

14. Kozlitina J, Garcia CK. Red blood cell size is inversely associated with leukocyte telomere length in a large multi- ethnic population. PLoS One 2012;7:e51046.

15. Patel KV, Semba RD, Ferrucci L, et al. Red cell distribution width and mortality in older adults: a metaanalysis. J Gerontol A Biol Sci Med Sci 2010;65:258-65.

16. Wu B, Lin S, Hao Z, et al. Proportion, risk factors and outcome of lacunar infarction: a hospital-based study in a Chinese population. Cerebrovasc Dis 2010;29:181-7.

17. The World Health Organization MONICA Project (monitoring trends and determinants in cardiovascular disease): a major international collaboration. WHO MONICA Project Principal Investigators. J Clin Epidemiol 1988;41:105-14.

18. Lippi G, Turcato G, Cervellin G, et al. Red blood cell distribution width in heart failure: A narrative review. World J Cardiol 2018;10:6-14.

19. Lu Z, Zhong N. Anemia. Internal Medicine. People's Medical Publishing House, 2nd ed, Beijing, 2008.

20. von Elm E, Altman DG, Egger M, et al. The Strengthening the Reporting of Observational Studies in Epidemiology (STROBE) statement: guidelines for reporting observational studies. Lancet 2007;370:1453-7.

21. Stroup DF, Berlin JA, Morton SC, et al. Meta-analysis of observational studies in epidemiology: a proposal for reporting. Meta-analysis Of Observational Studies in Epidemiology (MOOSE) group. JAMA 2000;283:2008-12.

22. Tian W, Xia X, Wang S, et al. Influence of red blood cell distribution on short-term functional outcome in patients with acute ischemic stroke and atrial fibrillation. Int J Clin Exp Med 2018;11:8392-401.

23. Duchnowski P, Hryniewiecki T, Kusmierczyk M, et al. Red cell distribution width is a prognostic marker of perioperative stroke in patients undergoing cardiac valve surgery. Interact Cardiovasc Thorac Surg 2017;25:925-9.

24. Kara H, Degirmenci S, Bayir A, et al. Red cell distribution width and neurological scoring systems in acute stroke patients. Neuropsychiatr Dis Treat 2015;11:733-9.

25. Lippi G, Targher G, Montagnana M, et al. Relation between red blood cell distribution width and inflammatory biomarkers in a large cohort of unselected outpatients. Arch Pathol Lab Med 2009;133:628-32.

26. Zurauskaite G, Meier M, Voegeli A, et al. Biological pathways underlying the association of red cell distribution width and adverse clinical outcome: Results of a prospective cohort study. PLoS One 2018;13:e0191280.

27. Hunziker S, Celi LA, Lee J, et al. Red cell distribution width improves the simplified acute physiology score for risk prediction in unselected critically ill patients. Critical Care 2012;16:R89. 
28. Lee KH, Cho JG, Park HW, et al. Role of Red Cell Distribution Width in the Relationship between Clinical Outcomes and Anticoagulation Response in Patients with Atrial Fibrillation. Chonnam Med J 2018;54:113-20.

29. Adamsson Eryd S, Borne Y, Melander O, et al. Red blood cell distribution width is associated with incidence of atrial fibrillation. J Intern Med 2014;275:84-92.

30. Ellingsen TS, Lappegard J, Skjelbakken T, et al. The association between red cell distribution width and venous thromboembolism is not explained by myocardial infarction, stroke, or cancer. Res Pract Thromb Haemost 2018;2:327-33.

31. Wang P, Wang Y, Li H, et al. Relationship between the red blood cell distribution width and risk of acute myocardial infarction. J Atheroscler Thromb 2015;22:21-6.

32. Duchnowski P, Szymanski P, Orlowska-Baranowska E, et al. Raised red cell distribution width as a prognostic marker in aortic valve replacement surgery. Kardiol Pol 2016;74:547-52.

33. Weymann A, Ali-Hasan-Al-Saegh S, Popov AF, et al.

Cite this article as: Wang L, Wang C, Wu S, Li Y, Guo W, Liu M. Red blood cell distribution width is associated with mortality after acute ischemic stroke: a cohort study and systematic review. Ann Transl Med 2020;8(4):81. doi: 10.21037/ atm.2019.12.142
Haematological indices as predictors of atrial fibrillation following isolated coronary artery bypass grafting, valvular surgery, or combined procedures: a systematic review with meta-analysis. Kardiol Pol 2018;76:107-18.

34. Perlstein TS, Weuve J, Pfeffer MA, et al. Red blood cell distribution width and mortality risk in a community-based prospective cohort. Arch Intern Med 2009;169:588-94.

35. Patel KV, Ferrucci L, Ershler WB, et al. Red blood cell distribution width and the risk of death in middle-aged and older adults. Arch Intern Med 2009;169:515-23.

36. Yao HM, Sun TW, Zhang XJ, et al. Red blood cell distribution width and long-term outcome in patients undergoing percutaneous coronary intervention in the drug-eluting stenting era: a two-year cohort study. PLoS One 2014;9:e94887.

37. Tonelli M, Sacks F, Arnold M, et al. Relation Between Red Blood Cell Distribution Width and Cardiovascular Event Rate in People With Coronary Disease. Circulation 2008;117:163-8. 


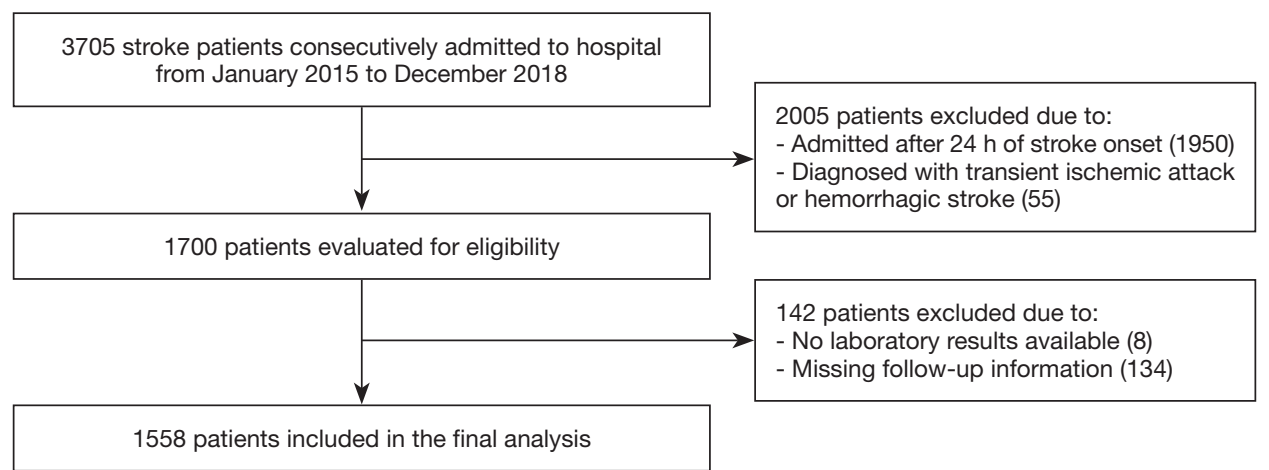

Figure S1 Flowchart for the selection process of eligible patients for cohort study.

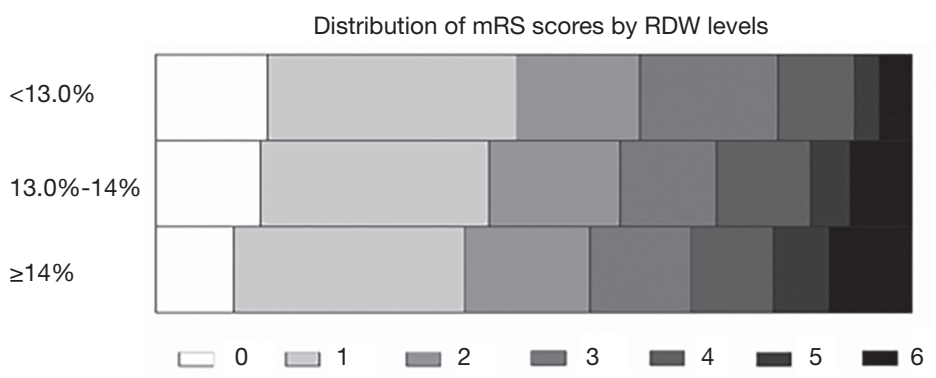

Figure S2 The distribution of modified Rankin Scale score grouped by red cell distribution width (RDW) values. mRS, modified Rankin Scale; RDW, red cell distribution width.

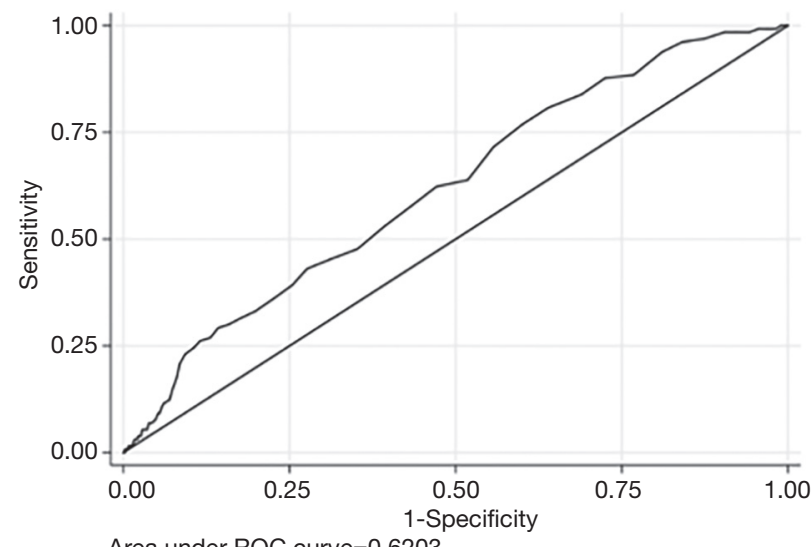

Figure S3 The relative operating characteristic (ROC) curve of red cell distribution width (RDW) values for predicting 3-month death. 

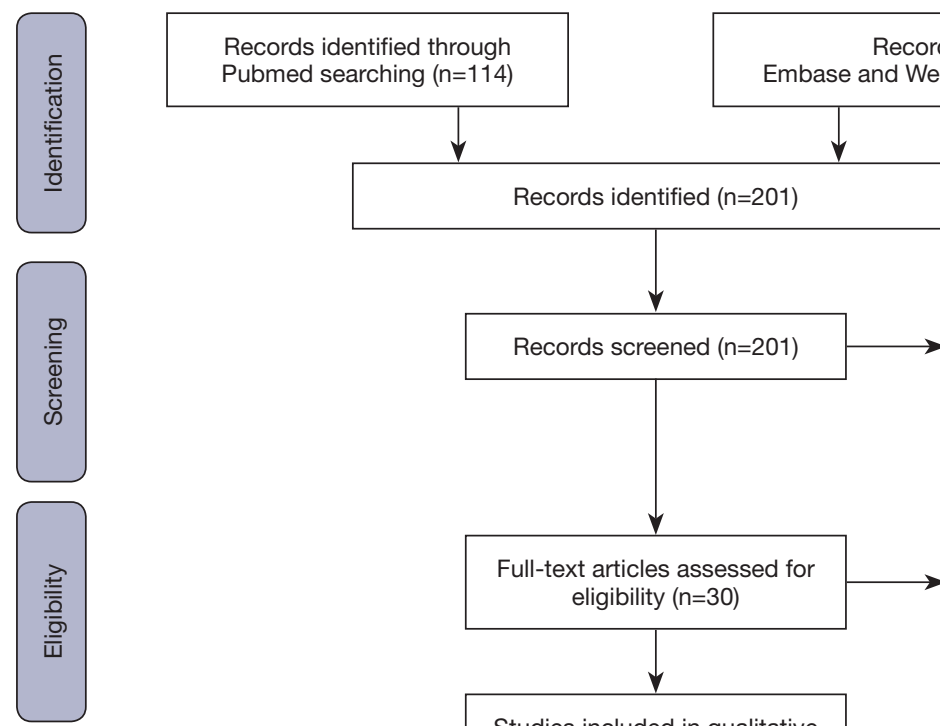

Pubmed searching $(n=114)$
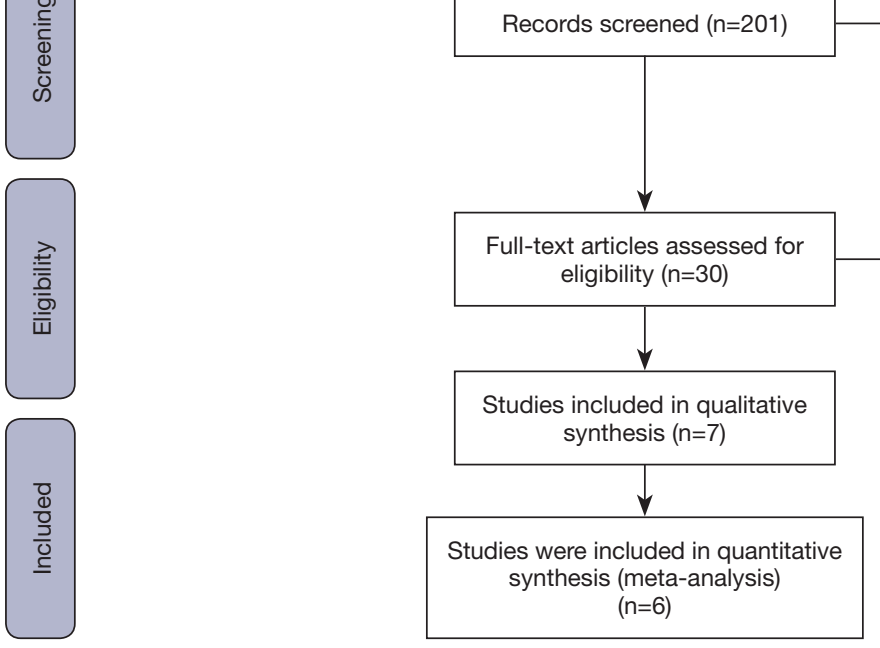

Figure S4 Flowchart of study selection process and the screening steps for systematic review.
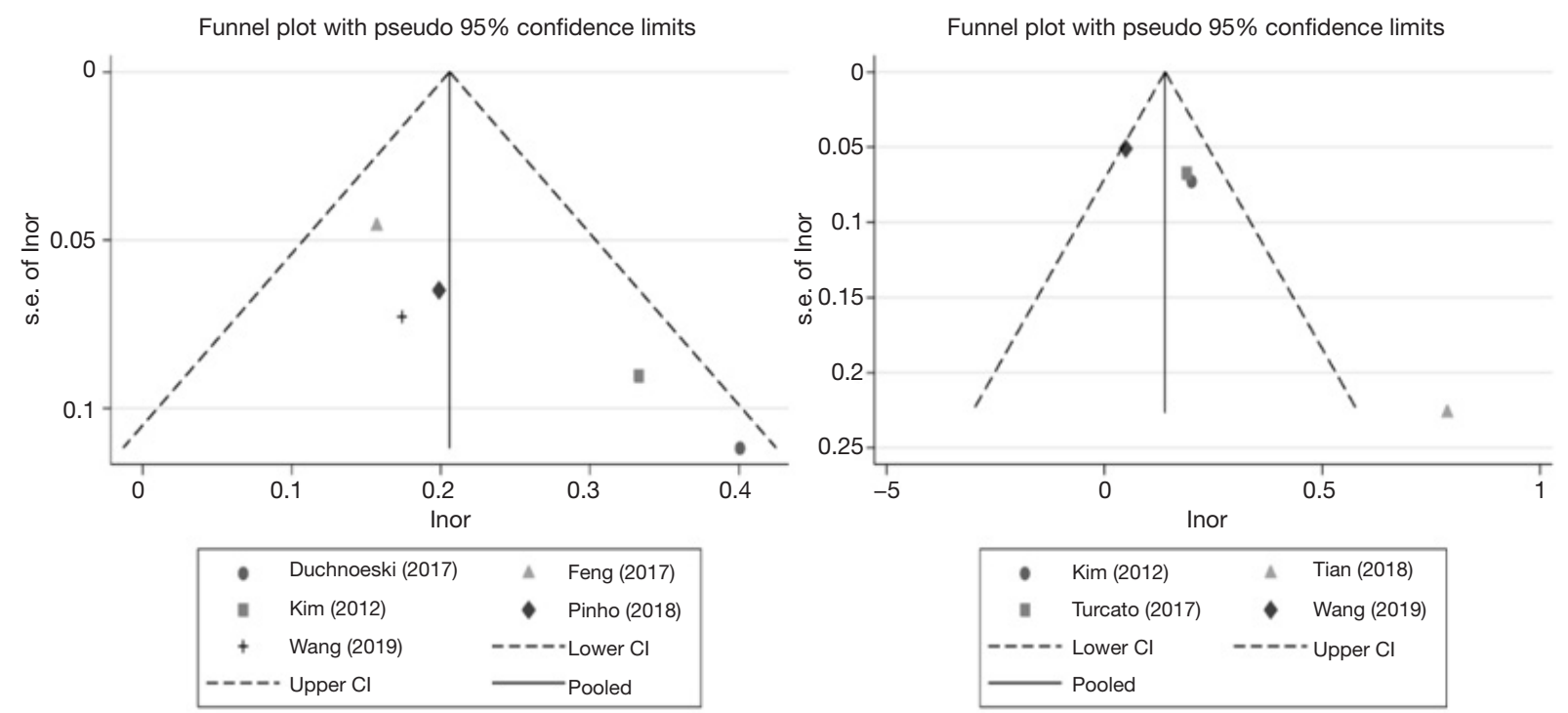

Figure S5 Funnel plot for red cell distribution width (RDW) predicting death (left) and poor outcome (right). 
Table S1 Baseline characteristics of included patients grouped by outcome at 3-month

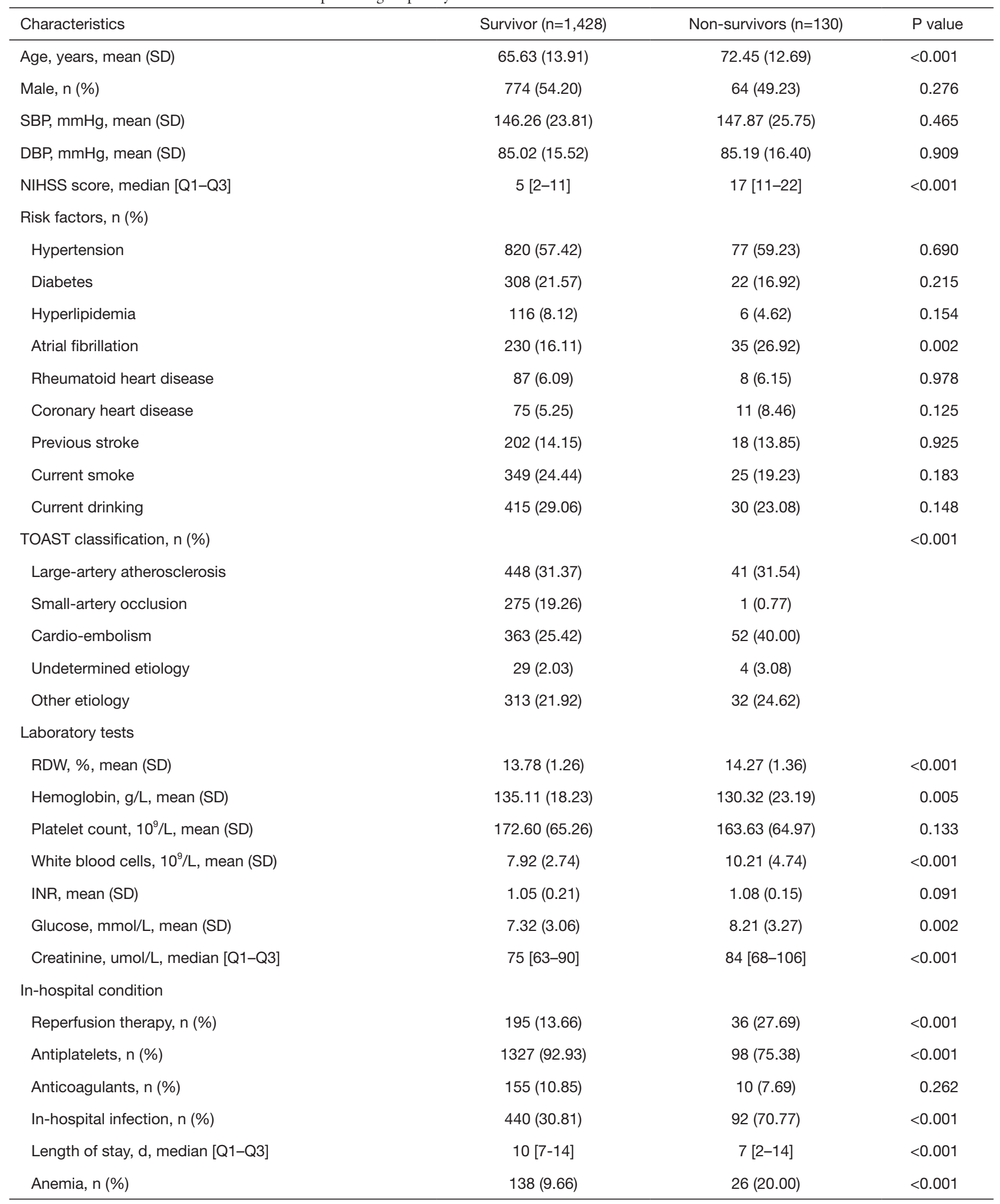

